\title{
Combining vector-phase coronagraphy with dual-beam polarimetry
}

\author{
Frans Snik ${ }^{a}$, Gilles Otten ${ }^{a}$, Matthew Kenworthy ${ }^{a}$, Dimitri Mawet ${ }^{b}$, Michael Escuti $^{c}$ \\ ${ }^{a}$ Sterrewacht Leiden, Universiteit Leiden, Niels Bohrweg 2, 2333 CA, Leiden, the Netherlands; \\ ${ }^{b}$ European Southern Observatory, Alonso de Cordova 3107, Vitacura, 763 0355, Santiago, Chile \\ ${ }^{c}$ North Carolina State University, Dept. of Electrical \& Computer Engineering, 432 Monteith, Campus Box 7914, \\ Raleigh, NC 27695-7914, USA.
}

\begin{abstract}
Utilizing the so-called vector phase of polarized light, both focal-plane coronagraphs (e.g. the Vector Vortex Coronagraph) and pupil-plane coronagraphs (e.g. the vector Apodizing Phase Plate) are powerful components for high-contrast imaging. These coronagraphs can be built and optimized with polarization techniques and liquid crystal technology, that enable patterning at the micron level and furnish broad-band performance. The contrast between the residual starlight and the (polarized) reflected light off exoplanets can be further bridged by incorporating sensitive, dual-beam imaging polarimetry. As vector-phase coronagraphs use polarizers to enhance their performance, we introduce optimally integrated solutions that combine advanced coronagraphy and polarimetry. For both the VVC and the VAPP we present polarization beam-splitting concepts, with polarization analyzers either behind or in front of the coronagraphic optics. We discuss design solutions for the implementation of polarization optics, and set the stage for a trade-off between the improvement of coronagraphic and polarimetric performance and the ensuing degradation on the high-contrast imaging performance due to wavefront errors.
\end{abstract}

Keywords: coronagraphy, polarimetry, vector phase, high-contrast imaging

\section{INTRODUCTION}

One of the most major and most exciting challenges in astronomy is to enable direct imaging and direct characterization of exoplanets, with the ultimate goal of photographing a Earth-like planet in the habitable zone of another star than the Sun, and analyzing the properties of its atmosphere and surface. A combination of advanced instrumentation and techniques is required to achieve this, as such planets are orders of magnitude fainter than their parent stars (contrasts of $\sim 10^{-5}$ down to $10^{-10}$ for a Sun-Earth equivalent in visible light), while their angular separation is small (usually measured in milli-arcsecond units). To provide sufficient angular separation (and photon flux for faint planets), we need large telescopes. High-contrast imaging with such telescopes relies on extreme adaptive optics systems to create PSFs with high Strehl ratios. But even for highly optimized adaptive optics systems the diffraction halo of the PSF is still much brighter than the planet signal. A large range of coronagraphic implementations ${ }^{1}$ have therefore been developed to suppress this halo. For this paper, we discern focal-plane coronagraphs that aim at blocking or nulling the on-axis starlight, and pupil-plane coronagraphs that modify the stellar PSF such that a dark hole is created in which one can search for planet signals. In particular, we focus on phase manipulation in the focal and/or pupil plane, such that starlight (locally) vanishes due to destructive interference.

For ground-based high-contrast systems, the coronagraphic PSF is generally still insufficient to furnish direct imaging of exoplanets, and therefore differential techniques have to be added to the mix. Current direct-imaging results rely heavily on Angular Differential Imaging, which utilizes sky rotation with respect to the quasi-static speckle halo. The new generation of dedicated high-contrast imaging instruments (like VLT/SPHERE and Gemini/GPI) can also distinguish planets from speckles by analyzing their spectral characteristics (Spectral Deconvolution Imaging through integral-field spectroscopy). However, both these techniques break down at a few $\lambda / D$ angular separation from the star, which is exactly where we need to search for rocky planets in the

email: snik@strw.leidenuniv.nl 
habitable zones of other stars. Therefore we need to develop more aggressive coronagraphs, but it is also highly opportune to invoke polarimetry ${ }^{2}$ as a differential technique. Reflected light off planets (or other circumstellar matter) is generally highly polarized ( $\sim 40 \%$ for Jupiter and Earth at quadrature), whereas the direct starlight is virtually unpolarized. Sensitive imaging polarimetry can therefore bridge the final orders of magnitude to reach an overall contrast that is sufficient to detect cold and old Jupiter-like planets at $\sim 10^{-8}$ contrast in visible light ${ }^{3}$ and even rocky planets with ELTs. ${ }^{4}$ Moreover, once a planet has been detected with sufficient S/N, the polarimetry can also be used to characterize that planet, and eventually detect signatures of habitibility and habitation (by whatever life-form). ${ }^{5,6}$

The success of any high-contrast instrument is dependent on its integration and optimization as a system. In this paper we introduce optical concepts for combining coronagraphy with polarimetry. Opportunities for highly integrated designs arise from the fact that the phase-manipulation of many coronagraphs can be implemented through the "vector phase" that operates on polarized components of light (see Sect. 2). In many cases, polarizers are introduced in the beam to improve the coronagraph performance. These polarizers can then of course also be used to measure polarization. We strive for designs with polarizing beam-splitters instead of regular polarizers, for the sake of photon-efficiency, but most of all to enable dual-beam polarimetry, which offers a significant contrast gain. ${ }^{4}$ Our goal is to inform upgrades to current high-contrast imaging polarimeters ${ }^{3,7}$ and to drive the design of instruments attached to future ground-based ${ }^{4}$ or space-based ${ }^{8}$ telescopes.

\section{THE VECTOR PHASE}

Phase manipulation in the classical sense is performed using shaped or corrugated optical surfaces. In addition to the fact that such optical components are often difficult to manufacture, they produce a phase pattern that is inherently chromatic in terms of fractions of waves. These issues limit coronagraphs that rely on classical phase manipulation. The Vortex Coronagraph consists of a continuous phase ramp around a central singularity on which the stellar PSF is centered, such that destructive interference between different sides of the PSF leads to a complete nulling of the on-axis starlight. This light is moved outside of the consecutive pupil plane and can be blocked there by a Lyot stop. The Vortex Coronagraph can be considered a generalization of the Four-Quadrant Phase Mask ${ }^{9}$ and the Eight-Octant Phase Mask. ${ }^{10}$ It is obvious that such a classical Vortex Coronagraph is a very complex optic (as it looks like a winding staircase), and that it is limited to narrow-band use. As for pupil-plane coronagraphs, the Apodizing Phase Plate ${ }^{11-13}$ impinges a particular pupil phase pattern such that the light in a D-shaped region next to the core of the ensuing PSF is moved to the other side of the PSF. Next to the already mentioned disadvantages of manufacturability and spectral bandwidth, this coronagraph has an obvious limitation if the exoplanet you're looking for happens to reside on the bright side of the PSF.

All these issues can be solved by invoking a different method of manipulating phase with spatial variation: through the so-called "vector phase" (a.k.a. "geometric phase" or "Pancharatnam-Berry phase"). The most useful implementation of this vector phase is through patterned half-wave retarders that operate upon circular polarization states. Regardless of the orientation of the fast axis of a half-wave plate, it flips the handedness of circular polarization. But the absolute phase of the emergent light is directly related to the axis orientation: a $180^{\circ}$ rotation induces a full $2 \pi$ phase shift. This phase shift is opposite for the two circular polarization states. ${ }^{14}$ One significant advantage of a vector-phase implementation is that phase ramps $>2 \pi$ are easily achieved by continuous rotation of the half-wave retarder axis orientation. So while the classical Vortex coronagraph necessarily contains a physical phase jump of $2 \pi$ or more, a Vector Vortex Coronagraph $\left(\mathrm{VVC}^{14-18}\right)$ is elegantly obtained with an azimuthal pattern of half-wave retarder orientations. The amount of $2 \pi$ phase ramps upon a full azimuthal revolution is known as the topological charge of the Vortex Coronagraph, and determines its coronagraphic performance and inner working angle. ${ }^{14,15}$ VVC masks can be manufactured using birefringent liquid crystal polymers ${ }^{14}$ or through patterned subwavelength gratings (form birefringence). ${ }^{19}$ Using liquid crystal patterning through a direct-writing system of the alignment layer ${ }^{20}$ virtually any phase pattern can be created with relative ease!

As the VVC is fully rotationally symmetric, the PSF for both circular polarization states is the same. This is not the case for the vector Apodizing Phase Plate, ${ }^{21,22}$ which antisymmetric pupil phase pattern generates mirror-symmetric PSFs for opposite circular polarizations. This means that after splitting of these circular 
polarizations (using a quarter-wave plate and a polarizing beam-splitter), the vAPP produces two PSFs with dark holes on either side, thereby solving one of the major limitations of the APP coronagraph.

Another major benefit of the vector phase is that it allows for achromatization, and thus enables broadband use of the coronagraphic device. Several techniques exist to create achromatic (half-wave) retarders: by combining different crystals with opposite dispersion of birefringence, by layering identical wave plates with different orientation (Pancharatnam principle), or with composite subwavelength structures. Liquid crystal vector-phase devices can be achromatized in similar ways, and, moreover, also the twisting nature of nematic liquid crystals can be used to one's advantage. By combining several layers of liquid crystal with different thicknesses, different amounts of twist, and potentially different amounts of birefringence dispersion (so-called Multi-Twist Retarders; $\mathrm{MTR}^{25}$ ), spectral bandwidths larger than an octave can be achieved. These liquid crystal layers are self-aligning, so they are perfectly adapted to create complex broad-band vector-phase patterns. ${ }^{23,24}$

\subsection{Leakage Terms}

The retardance of vector-phase coronagraphs is never perfectly half-wave, and offsets from half-wave retardance induce leakage terms. In the base of the two circular polarization states, such a retardance offset means that the flip of handedness from left- to right-handed (and vice versa) is incomplete, and that a fraction of the light $\left(\sin ^{2}\left(\frac{1}{2} \Delta \delta_{\mathrm{HW}}\right)\right)$ does not get the desired phase pattern imprinted on it, which for both the case of the VVC and the vAPP leads to a print-through of the Airy pattern of the original PSF. This leakage can be filtered by only transmitting one circular polarization (say, left-handed) to the vector-coronagraph, and filtering for the other one (right-handed) behind it. As true circular polarizers are not widely available, such circular polarization filters can be created by combining a regular linear polarizer with a quarter-wave plate (QWP) that converts linear polarization into circular. For the first filter, the linear polarizer comes first, and then the QWP at $45^{\circ}$, and for the second filter they are to be placed the other way around. For a configuration with crossed identical QWPs and crossed polarizers, the leakage terms pertaining to offsets of half-wave retardance disappear, even in the presence of offsets to the quarter-wave retardance. Such offsets create leakage terms of their own, but only of magnitude $\sin ^{4}\left(\frac{1}{2} \Delta \delta_{\mathrm{QW}}\right)$. Other even higher-order terms appear when the offsets are different for the two QWPs. Also the finite extinction ratios of the polarizers (see Sect. 5) and the alignment accuracy of all the polarization optics need to be factored in.

The vAPP already requires the implementation of one QWP and a polarizing beam-splitter (or a regular polarizer for a single-beam version). Therefore, all pertinent leakage terms need to be taken into account to assess its baseline performance. These leakage terms include both printing-though of the original PSF and mixing of the two modified PSFs. ${ }^{22}$

\section{SYSTEM DESIGNS FOR THE VECTOR VORTEX CORONAGRAPH}

In Fig. 1 we sketch systems designs hat incorporate the VVC coronagraph and polarimetry of increasing performance. The various optical elements are color-coded cf.:

coronagraphic optics

leakage filtering optics

polarimetric optics

Note that some optical elements can serve different purposes simultaneously.

Configuration 1A represents the most basic implementation of the VVC. It is easily implemented in the classical configuration of a Lyot coronagraph, with a Lyot stop behind it to block the diffracted light off the focal-plane mask. The pupil plane in front of the coronagraph can be used for apodization. ${ }^{26,27}$ This VVC implementation has been successfully commissioned on-sky. ${ }^{19,28,29}$ A multistage version of this set-up can be used to deal with pupil shapes with obscurations ${ }^{30}$ (e.g. due to the secondary mirror), which also provides opportunities for focal-plane wavefront sensing. ${ }^{31}$ 
$1 \mathrm{~A}$ regular VVC configuration

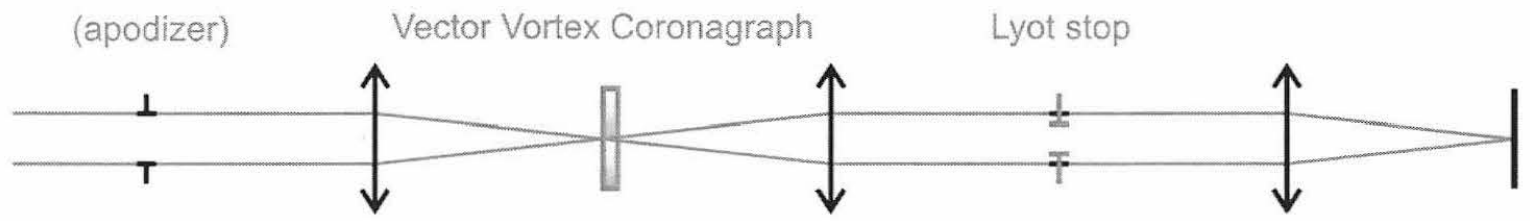

$1 \mathrm{~B}$ VVC with leakage filtering and single-beam polarimetry

pol. mod polarizerQWP

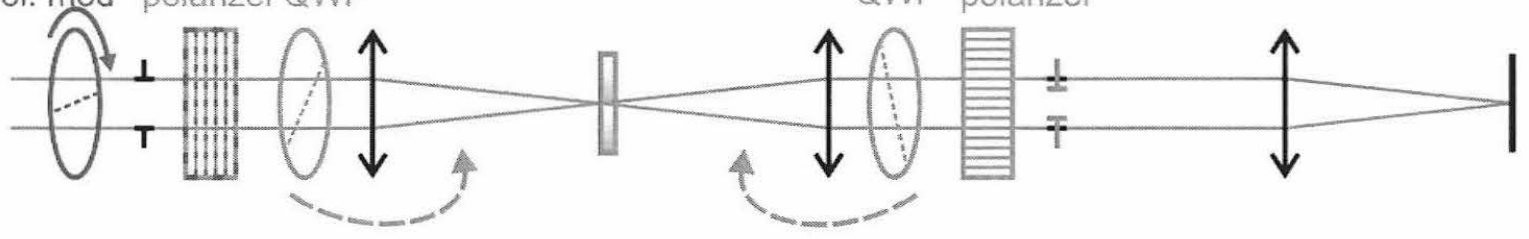

1C $2 x$ VVC with leakage filtering and $2 x$ single-beam polarimetry

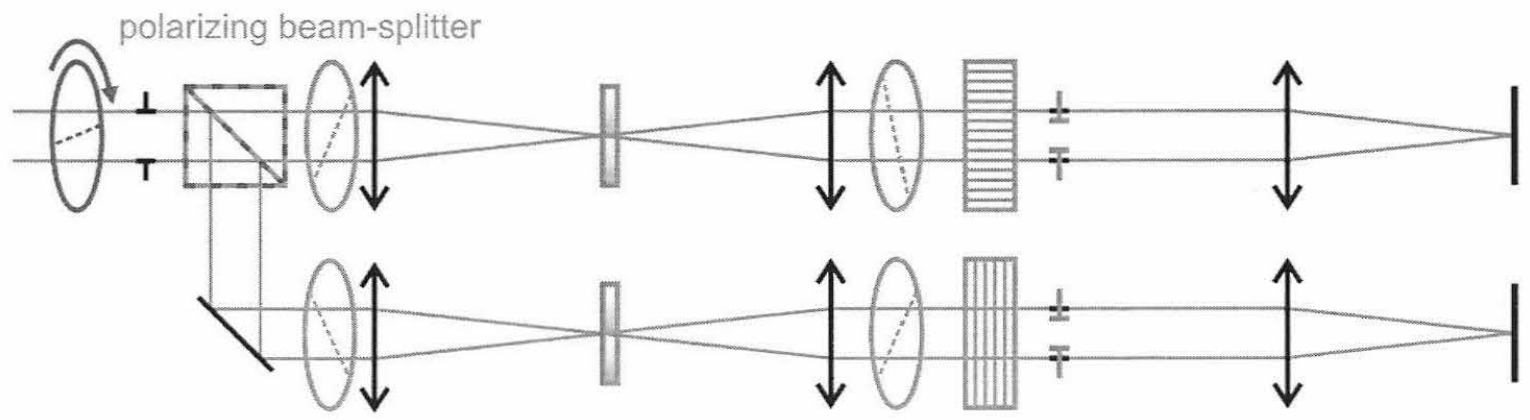

1D $1 \times$ VVC with dual-beam leakage filtering

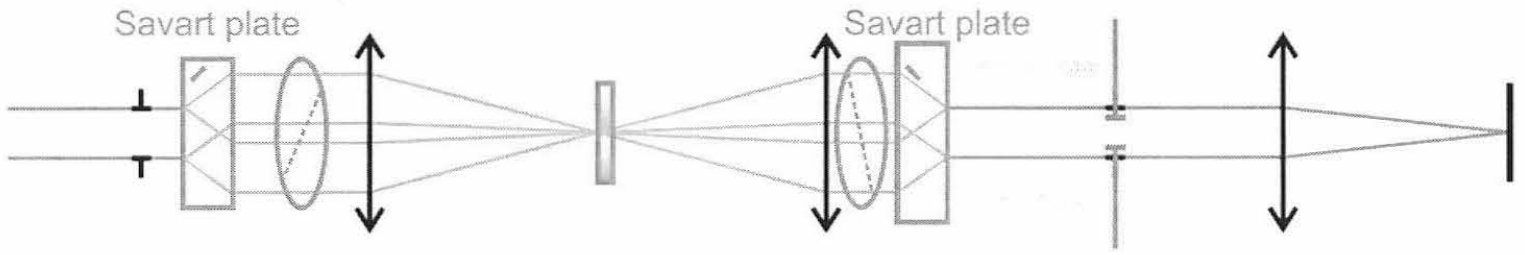




\section{E 1x VVC with leakage filtering and dual-beam polarimetry}

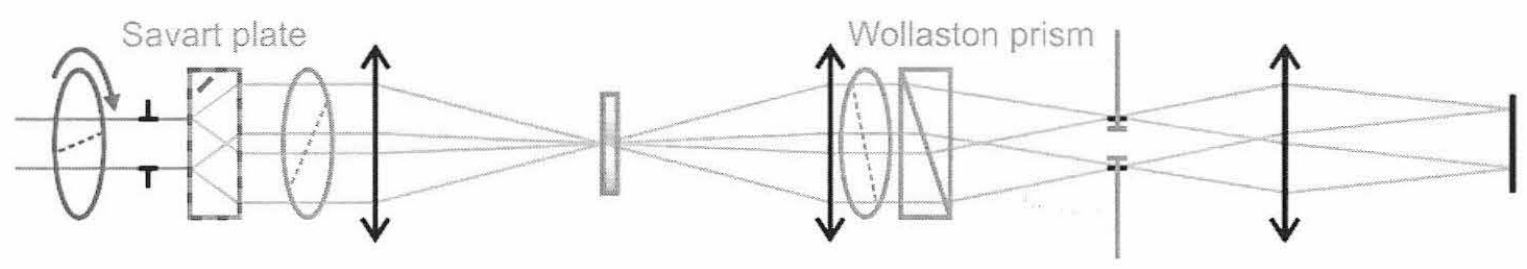

Figure 1. VVC design options including polarimetric implementations.

It is possible to implement polarimetry in this set-up completely independently from the coronagraphy. Any kind of polarimeter could be set up behind these coronagraphic optics. However, the VVC mask, being a patterned half-wave retarder, does rotate the measurable linear polarization as a function of field position. Polarization calibration of such a system is therefore even more intricate than it would normally be.

Because of the rotational symmetry of the VVC, the PSFs according to the two circular polarization states are identical. Splitting circular polarizations enables a complementary implementation for wavefront sensing by comparing the two PSFs. ${ }^{32}$ However, to filter leakage terms due to retardance offsets in the VVC mask, it is to be sandwiched in between linear polarizers and QWPs, as depicted in configuration $1 \mathrm{~B}$. The performance of polarization optics is usually optimal in the collimated beams on either side of the coronagraphic focal plane. But the re-imaging optics are likely to induce retardance, either due to mirror reflections at non-normal incidence or due to stress birefringence in lenses, which effectively reduces the purity of the circular polarization state and thus reduces the suppression of leakage terms. Moreover, mirrors have the fundamental property of flipping the handedness of circular polarization. For beams with large focal ratios, the leakage-filtering polarizers and QWPs could therefore also be located in the converging/expanding beam on either side of the VVC mask.

With the inclusion of a linear polarizer arises the opportunity to measure polarization, before it gets modified by the coronagraph. In combination with a polarization modulator (e.g. a rotating wave plate of a switching liquid crystal $^{2}$ ), several polarization states (e.g. $I \pm Q, I \pm U$ ) can be sequentially converted to the linear polarization that the first polarizer filters, and thus a measure of the fractional Stokes parameters $(Q / I, U / I)$ can be obtained. However, such a single-beam polarimetric implementation is notoriously susceptible to any temporal variations that are not due to the polarization modulation, most prominently those induced by atmospheric seeing. To still attain high polarimetric sensitivity with a ground-based single-beam system, modulation frequencies of $\sim 1 \mathrm{kHz}$ or higher are required. ${ }^{3}$

Another way to achieve high polarimetric sensitivity, even with slow(er) modulation, is to implement a dual-beam system that simultaneously records orthogonal polarization states, in combination with temporal modulation. The imposed measurement redundancy permits cancellation of differential effects both in the temporal domain as in the spatial domain (e.g. transmission differences, limited gain table calibration, differential aberrations). ${ }^{2}$ One solution for implementing dual-beam polarimetry for a VVC coronagraph system is presented in configuration $\mathbf{1 C}$, which simply replaces the first polarizer with a polarizing beam-splitter that feeds two separate but identical systems. ${ }^{15,33}$ Note that folding mirrors tend to reduce the polarization property of circular polarization, regardless of their orientation, and, morover, flip handedness. A harmless implementation of such a mirror is therefore directly behind the polarization beam-splitter, such that the split linear polarization coincides with the $s / p$ direction of the mirror. Such a set-up introduces the opportunity for polarization-dependent wavefront correction by implementing deformable mirrors in each of the separate beams. ${ }^{15}$ Polarization-dependency of wavefront errors limit the coronagraphic performance of space-based systems that are required to provide raw contrasts as extreme as $10^{-9}$ (which is impossible to attain from the ground because of atmospheric seeing which cannot be perfectly corrected because of finite adaptive optics loop speed). So, whereas polarimetry for ground-based high-contrast systems is primarily a contrast-enhancing tool, for a space-based telescope it can be directly used for characterization of exoplanets and other circumstellar matter. 
To reduce the complexity of the separate dual-beam system, configuration $1 \mathrm{D}$ presents a first step towards feeding two leakage-filtered beams through a single VVC mask down to a single detector. A polarizing beamsplitter like a Savart plate first laterally splits the collimated beam, whilst maintaining the angular properties. The re-imaging lens then focuses both separate beams onto the VVC mask. An identical polarizing beam-splitter in the collimated beam after the mask then recombines the beams, and create a single PSF. Because the leakage terms are deviated in the opposite direction, they are easily caught off by the Lyot stop. In such a set-up ${ }^{10}$ the splitting and recombination of the polarization components of the beam occur in a coherent fashion. This means that the first polarizing beam-splitter does not act as a polarization analyzer, as the two linear polarization components can recombine again to the incident polarization state. Polarimetry could therefore be implemented behind the coronagraphic optics train, but that will act as a complicated retarder element with properties that depend on the location in the field-of-view. These issues could be aggravated by the occurrence of fringes, as the set-up is essentially a Michelson interferometer.

In configuration $1 \mathrm{E}$ these issues are solved, as the beams are not recombined: a Wollaston prism crosses the beams at the location of the Lyot stop, such that the Lyot stop still catches both the diffracted light and the leakage terms. The two beams are already essentially split by the Savart plate before the VVC mask, and therefore also furnish dual-beam polarimetry together with a polarization modulator upstream.

\section{SYSTEM DESIGNS FOR THE VECTOR APODIZING PHASE PLATE}

The basic implementation of the VAPP is sketched in configuration 2A in Fig. 2. The mask itself is located in a pupil plane, and after it the beam is split according to circular polarization states by a combination of a QWP and a Wollaston prism. A camera lens then produces two PSFs with dark holes on opposite sides. ${ }^{21,22}$ The purest system in terms of optical performance is obtained when the vAPP mask, the QWP and the Wollaston are put together as closely as possible, such that both the coronagraphic phase pattern and the angular splitting due to the Wollaston occur as close as possible to the true pupil-plane location. Obviously, also other implementations for the polarizing beam-splitting could be considered, and even a single-beam system with a regular polarizer could have its benefits.

A first combination with polarimetry is obtained by turning the vAPP assembly into a polarizing beam-splitter for linear polarization by adding one more QWP in front (configuration 2B). The first QWP then converts the linear polarization directions that are to be splitted $\left(0 / 90^{\circ}\right)$ into circular polarizations with opposite handedness. The vAPP mask, being a patterned half-wave retarder, merely flips the handedness of these circular polarization states, regardless of the local orientation pattern. The second QWP then converts these circular polarization back to $0 / 90^{\circ}$, which are consecutively split by the Wollaston. A polarization modulator in front of this beam-splitter then completes the polarimeter. It needs to be emphasized that this system is not a dual-beam polarimeter, as the two PSFs are very different by design. But any circumstellar object in one of the two dark holes can be probed with single-beam polarimetry, which can be very sensitive in case of fast modulation. ${ }^{3}$ Any residual polarimetric degradation due to seeing may be partially correctable by using the simultaneous information from the two stellar PSF cores (provided those are not saturated).

A next step (configuration $\mathbf{2 C}$ ) involves the incorporation of leakage-term filtering. This is enabled by moving the Wollaston prism in front of the first QWP and the vAPP mask. The filtering itself then needs to occur near the focal plane, where the beams are fully split. A compound linear polarizer at $0^{\circ}$ and $90^{\circ}$ can then selectively take out the leakage terms. The splitting by the Wollaston before the vAPP mask invariantly leads to a laterally sheared pupil at the position of the vAPP mask. This can accommodated by shrinking the mask size such that it is fully illuminated by both beams, obviously at the expense of light loss. To prevent this, a combination of two Wollaston prisms at a predetermined separation can generate split beams that exactly cross at the location of the vAPP mask (configuration 2D).

Again, these solutions only enable single-beam polarimetry for each of the PSFs. To produce two beams for each PSF for dual-beam polarimetry, another Wollaston prism and another QWP need to be implemented in front of the vAPP assembly (configuration $2 \mathrm{E}$ ). This Wollaston prism splits the beam in the other dimension (out of the plane in Fig. 2). The split linear polarizations $\left(90 / 0^{\circ}\right)$ are converted into circular by the first QWP. In turn, these circular polarization states are split into perpendicular linear polarization states by the second 
$2 \mathrm{~A}$

regular vAPP configuration

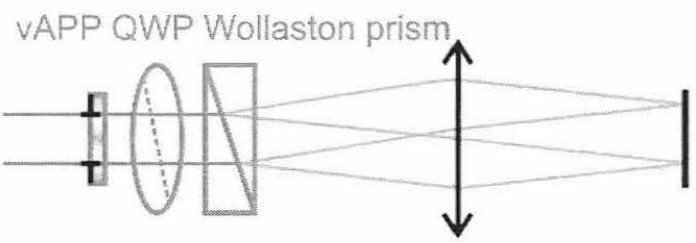

$2 B \quad$ vAPP with $2 x$ single-beam polarimetry

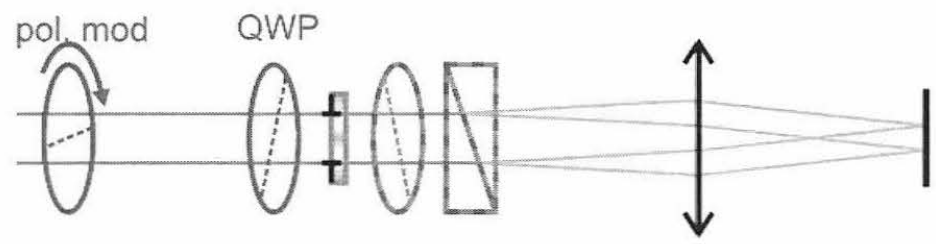

$2 \mathrm{C}$ vAPP with leakage filtering and $2 \mathrm{x}$ single-beam polarimetry, v1

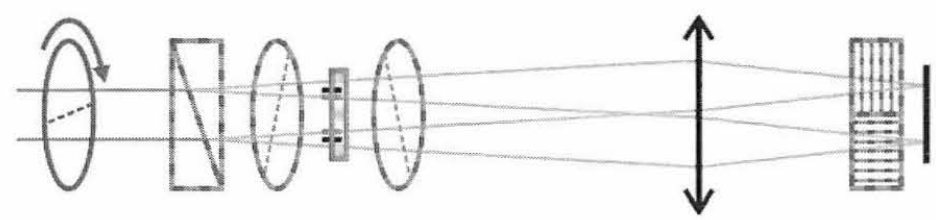

$2 \mathrm{D}$ vAPP with leakage filtering and $2 \mathrm{x}$ single-beam polarimetry, v2

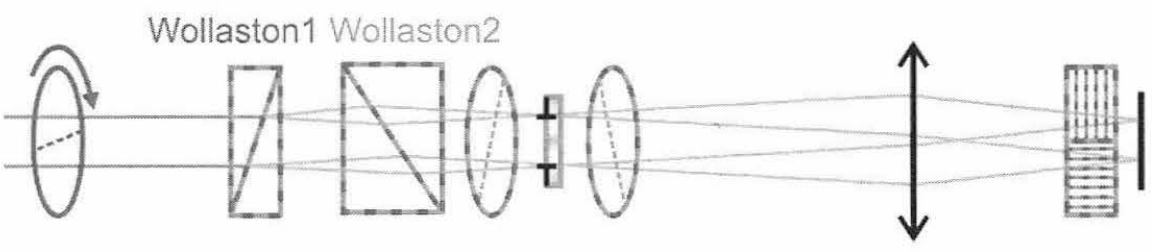




\title{
2E vAPP with leakage filtering and $2 x$ dual-beam polarimetry, v1
}

\author{
Wollaston1 Wollaston2

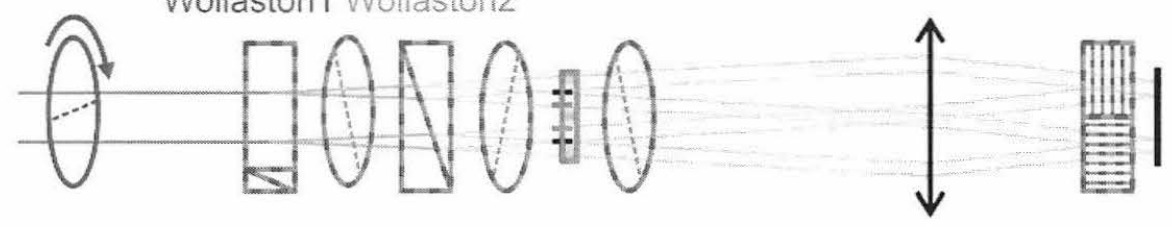

\section{$2 \mathrm{~F}$ vAPP with leakage filtering and $2 x$ dual-beam polarimetry, v2}

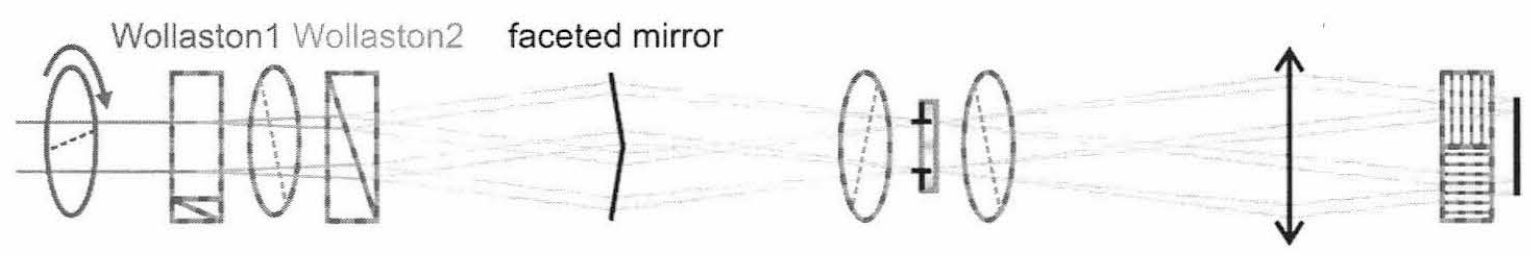

Figure 2. vAPP design options including polarimetric implementations.

Wollaston, and finally converted into circular polarization states to feed the vAPP mask plus leakage filtering elements. Again, this set-up yields displaced pupil images (four in this case) at the location of the vAPP mask, which necessitates a shrinking of the mask. To cross all four beams at the vAPP mask, both Wollaston prisms need to be moved upstream, and the four beams are to be folded back by a faceted mirror (configuration 2F). Unfortunately, the four beams do not have unique polarization states, so a solution with only polarization optics cannot be found.

\section{DESIGN OPTIONS FOR THE POLARIZATION OPTICS}

The detailed design of a high-contrast imaging system that incorporates coronagraphy and polarimetry is necessarily subject to a complex trade-off: the polarization optics add to the wavefront error budget, and all optical components in front of the polarimeter in one way or the other degrade the polarimetric performance. In general, the adaptive-optics system can deal with phase error conjugate to a pupil plane. Any amplitude aberrations lead to degraded coronagraphic performance, and out-of-pupil phase aberrations are converted into chromatic amplitude errors by Fresnel propagation effects. Therefore, it is preferable to locate as many of the polarization optics in the concepts presented above as possible close to the pupil plane. Fortunately, as the field-of-view of high-contrast imaging instrumentation is very small, collimated beams are usually almost in their entirety conjugate to the pupil. Moreover, deformable mirrors correct wave front errors with a $1 / \lambda$ term, and therefore the dispersion of transmissive optics can create chromatic phase terms that are uncorrectable. Requirements on wavefront errors are also lenient for optics in a focal plane, which makes that the leakage-filtering polarizer in configurations $2 \mathrm{C}-\mathrm{F}$ should be moved as close as possible to the detector.

The two split beams go through different parts of the subsequent optics, and hence they will accrue a slightly different wavefront error. This means that the adaptive optics cannot optimally correct both PSFs, even in the absence of polarization aberrations. That warrants beam-splitting as far downstream as possible, while for the polarimetry the polarization measurement should occur as much upstream as possible. However, polarization modulation and polarization can be split, and the intermediate optics do not degrade the polarimetry as long as the linear polarization directions that are to be analyzed are the eigenvectors of the optical system in between, ${ }^{2}$ e.g. corresponding to the $s$ and $p$ directions of mirrors. 
We provide a (non-exhaustive) overview of options for the implementation of polarization optics, which trade-off parameters are summarized in Tab. 1.

\subsection{Polarizers and Polarizing Beam-splitters}

The polarizers with the highest extinction ratios are based on birefringent crystals. Both a Wollaston prism and a Savart plate generally have a good polarization and optical performance in a collimated beam, although in both cases there are subtle optical differences between the two beams: for a Wollaston this is caused by splitting on an inclined surface, and inside both a Wollaston and a Savart the two beams see a different refractive index. A single Savart plate only deviates one beam, so it takes a double Savart plate to constitute a mostly symmetric system. The amount of splitting for these polarizing beam-splitters depends on the amount of birefringence, which is subject to chromatic dispersion. In configuration 1D this dispersion is fully compensated, and for configuration $1 \mathrm{E}$ optimal solutions can be found by making the Savart and the Wollaston out of the same material. It is usually a bad idea to put birefringent crystal optics in a converging beam, as the extinction ratio is then greatly reduced, and they induce specific optical aberrations like astigmatism (in addition to spherochromatism).

For large amounts of splitting, the material of choice in the visible range up to $2 \mu \mathrm{m}$ is calcite. This material can only be obtained from mining, and large chunks are difficult to procure. Moreover, crystal impurities can lead to wavefront errors. A calcite Savart plate induces a lateral splitting of about one tenth of the length of the overall plate. For fully separating a $1 \mathrm{~cm}$ pupil, it therefore takes a $10-\mathrm{cm}$ slab of calcite. An alternative lateral splitting can be devised with two Wollaston prisms with a certain amount of longitudinal separation. Or one can adopt a folding strategy using polarizing beam-splitter cubes. ${ }^{10}$

However, cube beam-splitters based on dielectric splitting coatings generally have limited extinction ratio and limited spectral bandwidth. Recently, polarizing cubes based on wire-grid polarizers have become available, which offer good performance for the transmitted beam, while the reflected beam may need to be "cleaned up" using a second wire-grid polarizer under normal incidence. For any kind of cube beam-splitter, much of the wavefront error can be controlled during manufacturing.

For regular polarizers, wiregrid technology now outperforms any kind of dichroic polymer polarizer in terms of extinction ratio and spectral bandwidth. Thanks to modern lithography, wiregrid polarizers can now be built for wavelength ranges down to the UV.

Table 1. Trade-off parameters for polarization optics.

\begin{tabular}{|c|c|c|c|c|}
\hline Component & Ext. Ratio & Wav. Range & Inc. Angle & WFE \\
\hline $\begin{array}{l}\text { dichroic sheet polarizer } \\
\text { wiregrid polarizer }\end{array}$ & $\begin{array}{l}+/- \\
+\end{array}$ & $\begin{array}{l}+/- \\
++\end{array}$ & $\begin{array}{l}+ \\
+\end{array}$ & + \\
\hline Glan prism & ++ & + & - & $+/-$ \\
\hline cube beam-splitter, dielectric coating & $+/-$ & $+/-$ & + & + \\
\hline cube beam-splitter, wiregrid coating & + & ++ & + & + \\
\hline Wollaston prism & + & + & $+/-$ & $+/-$ \\
\hline Savart plate & + & + & - & $+/-$ \\
\hline Component & Retardance & Wav. Range & Inc. Angle & WFE \\
\hline zero-order polymer & $+/-$ & $+/-$ & + & - \\
\hline quasi-zero order quartz & + & $+/-$ & - & + \\
\hline "achromatic" quartz $+\mathrm{MgF}_{2}$ & + & + & - & + \\
\hline Pancharatnam polymer & + & + & $+/-$ & - \\
\hline "super-achromatic" quartz $+\mathrm{MgF}_{2}$ & + & ++ & - & + \\
\hline liquid crystal MTR & $+/-$ & + & + & $+/-$ \\
\hline Fresnel rhomb & ++ & ++ & - & + \\
\hline form birefringence & + & + & $+/-$ & $+1-$ \\
\hline
\end{tabular}




\subsection{Achromatic Wave Plates}

The title of this subsection appears a contradictio in terminis, but various techniques have been developed to yield retarders that are close to half-wave or quarter-wave over a large spectral range. It is clear that these offsets from both half-wave and quarter-wave retardance are to be controlled to suppress leakage terms, as explained in subsection 2.1. Classical "achromatic" wave plates are obtained from the combination of two crystals (e.g. quartz and $\mathrm{MgF}_{2}$ ) that compensate each others' retardance dispersion over some range. Pancharatnam wave plates offer large spectral bandwidth by stacking an odd number identical wave plates at different orientations. The combination of these two techniques yields "superachromatic" wave plates, which offer a maximum retardance offset of $\sim 4^{\circ}$ over a spectral bandwidth larger than two octaves. Recently, liquid crystal MTR ${ }^{25}$ technology has been developed to offer even more degrees of freedom to achromatize waveplates, and can meet and exceed the performance of Pancharatnam plates ${ }^{22}$ in addition to its inherent patterning capability. Form birefringence, which is also used to create VVC coronagraphs, ${ }^{19}$ also offers good opportunities for creating achromatized wave plates in the IR.

All these wave plates do generally suffer from wavefront errors. Regular crystals can be polished well, but plates based on polymers or liquid crystals may prove difficult to create with high optical quality. Still, the best broad-band retarder with potentially excellent optical properties is the Fresnel rhomb. Unfortunately, it deviates the beam, which complicates the optical design, and limits its use as a rotating polarization modulator.

\subsection{Incidence Angle Effects}

Many of the solutions in Figs. 1 and 2 rely on angular splitting of collimated and converging beams. Therefore, additional polarization effects, and hence degraded performance of the vector-phase coronagraphy is to be expected. Non-normal incidence at any dielectric surface modifies the circular polarization state into an elliptical one according to the Fresnel equations. This reduction of polarization purity of the circular polarizations reduces the effectivity of the leakage-term filtering, but even for a series of air-glass interfaces at $10^{\circ}$ incidence angle this is a negligible effect (factor of 0.9999). Fold mirrors could cause more severe issues, as their reflectivity is different for the $s, p$ linear polarization states, and they invariantly decrease the purity of $100 \%$ circularly polarized states (in addition to flipping handedness).

Another effect to take into account is that the effective retardance change with incidence angle, and also with the relative orientation of that angle with respect to the retarder's fast axis, which is a strong effect for classical crystal optics. A Berreman calculus model for a 3TR achromatized liquid crystal device shows that retardance offsets up to $1.5^{\circ}$ can be expected for an incidence angle of $10^{\circ}$. While this is a significant effect, it is smaller that the inherent deviations from half-wave or quarter-wave retardance for such achromatized liquid crystal devices.

Finally, also the geometrical foreshortening of $\sim 1.5 \%$ due to a $10^{\circ}$ incidence angle can be safely neglected.

\section{OUTLOOK}

New high-contrast imaging instruments or upgrades to existing instruments can significantly profit from novel liquid crystal technology to create complex phase patterns for advanced coronagraphs that can operate over large $(\gg 10 \%)$ spectral bandwidths. In addition to the VVC and vAPP coronagraphs, many other other phasemanipulating coronagraph designs could benefit from this manufacturing technique. By sandwiching a patterned liquid crystal retarder between polarizers, also complex amplitude apodization can be accomplished. In general, extreme, hybridized coronagraphic solutions with phase/amplitude patterning in both pupil plane and focal plane can now be envisaged.

Moreover, the polarization techniques that are used to optimize the coronagraphic performance, can also be optimally used to implement polarimetry, which furnishes additional contrast enhancement and unique diagnostics of exoplanet atmosphere/surface properties. The integration of the various modalities of high-contrast instruments demands a systems approach that involves complex trade-offs. In addition to the coronagraphy and polarimetry that are considered in this paper, telescope performance, adaptive optics performance, integral-field spectroscopy, and data reduction need to be considered as well. The ultimate goal of direct imaging and direct characterization of a rocky planet in the habitable zone of another star than the Sun will only be possible by adopting an integrated view of the end-to-end system. 


\section{REFERENCES}

[1] D. Mawet, L. Pueyo, P. Lawson, L. Mugnier, W. Traub, et al., "Review of small-angle coronagraphic techniques in the wake of groundbased second-generation adaptive optics systems," in Society of Photo-Optical Instrumentation Engineers (SPIE) Conference Series, Society of Photo-Optical Instrumentation Engineers (SPIE) Conference Series 8442, Sept. 2012.

[2] F. Snik and C. U. Keller, Astronomical Polarimetry: Polarized Views of Stars and Planets, p. 175. 2013.

[3] C. Thalmann, H. M. Schmid, A. Boccaletti, D. Mouillet, K. Dohlen, R. Roelfsema, M. Carbillet, D. Gisler, J.-L. Beuzit, M. Feldt, R. Gratton, F. Joos, C. U. Keller, J. Kragt, J. H. Pragt, P. Puget, F. Rigal, F. Snik, R. Waters, and F. Wildi, "SPHERE ZIMPOL: overview and performance simulation," in Society of Photo-Optical Instrumentation Engineers (SPIE) Conference Series, Society of Photo-Optical Instrumentation Engineers (SPIE) Conference Series 7014, Aug. 2008.

[4] C. U. Keller, H. M. Schmid, L. B. Venema, H. Hanenburg, R. Jager, M. Kasper, P. Martinez, F. Rigal, M. Rodenhuis, R. Roelfsema, F. Snik, C. Verinaud, and N. Yaitskova, "EPOL: the exoplanet polarimeter for EPICS at the E-ELT," in Society of Photo-Optical Instrumentation Engineers (SPIE) Conference Series, Society of Photo-Optical Instrumentation Engineers (SPIE) Conference Series 7735, July 2010.

[5] D. M. Stam, "Spectropolarimetric signatures of Earth-like extrasolar planets," A\&A 482, pp. 989-1007, May 2008.

[6] T. Karalidi, D. M. Stam, and J. W. Hovenier, "Looking for the rainbow on exoplanets covered by liquid and icy water clouds," $A \& A$ 548, p. A90, Dec. 2012

[7] S. J. Wiktorowicz, M. Millar-Blanchaer, M. D. Perrin, J. R. Graham, S. J. Thomas, D. Dillon, M. P. Fitzgerald, J. Maire, B. A. Macintosh, and S. J. Goodsell, "Polarimetric performance of the Gemini Planet Imager," in Society of Photo-Optical Instrumentation Engineers (SPIE) Conference Series, Society of Photo-Optical Instrumentation Engineers (SPIE) Conference Series 8446, Sept. 2012.

[8] A. Boccaletti, J. Schneider, W. Traub, P.-O. Lagage, D. Stam, R. Gratton, J. Trauger, K. Cahoy, F. Snik, P. Baudoz, R. Galicher, J.-M. Reess, D. Mawet, J.-C. Augereau, J. Patience, M. Kuchner, M. Wyatt, E. Pantin, A.-L. Maire, C. Vérinaud, S. Ronayette, D. Dubreuil, M. Min, M. Rodenhuis, D. Mesa, R. Belikov, O. Guyon, M. Tamura, N. Murakami, and I. M. Beerer, "SPICES: spectro-polarimetric imaging and characterization of exoplanetary systems. From planetary disks to nearby Super Earths," Experimental Astronomy 34, pp. 355-384, Oct. 2012.

[9] D. Mawet, P. Riaud, J. Baudrand, P. Baudoz, A. Boccaletti, O. Dupuis, and D. Rouan, "The four-quadrant phase-mask coronagraph: white light laboratory results with an achromatic device," A\&A 448, pp. 801-808, Mar. 2006.

[10] N. Murakami, J. Nishikawa, K. Yokochi, M. Tamura, N. Baba, and L. Abe, "Achromatic Eight-octant Phase-mask Coronagraph using Photonic Crystal," ApJ 714, pp. 772-777, May 2010.

[11] M. A. Kenworthy, J. L. Codona, P. M. Hinz, J. R. P. Angel, A. Heinze, and S. Sivanandam, "First On-Sky High-Contrast Imaging with an Apodizing Phase Plate," ApJ 660, pp. 762-769, May 2007.

[12] M. A. Kenworthy, S. P. Quanz, M. R. Meyer, M. E. Kasper, R. Lenzen, J. L. Codona, J. H. Girard, and P. M. Hinz, "An apodizing phase plate coronagraph for VLT/NACO," in Society of Photo-Optical Instrumentation Engineers (SPIE) Conference Series, Society of Photo-Optical Instrumentation Engineers (SPIE) Conference Series 7735, July 2010.

[13] S. P. Quanz, M. R. Meyer, M. A. Kenworthy, J. H. V. Girard, M. Kasper, A.-M. Lagrange, D. Apai, A. Boccaletti, M. Bonnefoy, G. Chauvin, P. M. Hinz, and R. Lenzen, "First Results from Very Large Telescope NACO Apodizing Phase Plate: $4 \mu \mathrm{m}$ Images of The Exoplanet $\beta$ Pictoris b," ApJL 722, pp. L49-L53, Oct. 2010.

[14] D. Mawet, E. Serabyn, K. Liewer, C. Hanot, S. McEldowney, D. Shemo, and N. O'Brien, "Optical Vectorial Vortex Coronagraphs using Liquid Crystal Polymers: theory, manufacturing and laboratory demonstration," Optics Express 17, pp. 1902-1918, Feb. 2009.

[15] D. Mawet, L. Pueyo, D. Moody, J. Krist, and E. Serabyn, "The Vector Vortex Coronagraph: sensitivity to central obscuration, loworder aberrations, chromaticism, and polarization," in Society of Photo-Optical Instrumentation Engineers (SPIE) Conference Series, Society of Photo-Optical Instrumentation Engineers (SPIE) Conference Series 7739, July 2010.

[16] D. Mawet, E. Serabyn, K. Liewer, R. Burruss, J. Hickey, and D. Shemo, "The Vector Vortex Coronagraph: Laboratory Results and First Light at Palomar Observatory," ApJ 709, pp. 53-57, Jan. 2010.

[17] D. Mawet, N. Murakami, C. Delacroix, E. Serabyn, O. Absil, et al., "Taking the vector vortex coronagraph to the next level for groundand space-based exoplanet imaging instruments: review of technology developments in the USA, Japan, and Europe," in Society of Photo-Optical Instrumentation Engineers (SPIE) Conference Series, Society of Photo-Optical Instrumentation Engineers (SPIE) Conference Series 8151, Sept. 2011.

[18] E. Serabyn, J. Trauger, D. Moody, D. Mawet, K. Liewer, J. Krist, and B. Kern, "High-contrast imaging results with the vortex coronagraph," in Society of Photo-Optical Instrumentation Engineers (SPIE) Conference Series, Society of Photo-Optical Instrumentation Engineers (SPIE) Conference Series 8864, Sept. 2013.

[19] D. Mawet, O. Absil, C. Delacroix, J. H. Girard, J. Milli, J. O'Neal, P. Baudoz, A. Boccaletti, P. Bourget, V. Christiaens, P. Forsberg. F. Gonte, S. Habraken, C. Hanot, M. Karlsson, M. Kasper, J.-L. Lizon, K. Muzic, R. Olivier, E. Peña, N. Slusarenko, L. E. TacconiGarman, and J. Surdej. "L'-band AGPM vector vortex coronagraph's first light on VLT/NACO. Discovery of a late-type companion at two beamwidths from an F0V star," A\&A 552, p. L13, Apr. 2013.

[20] M. N. Miskiewicz and M. J. Escuti, "Direct-writing of complex liquid crystal patterns," Opt. Express 22, pp. 12691-12706, May 2014.

[21] F. Snik, G. Otten, M. Kenworthy, M. Miskiewicz, M. Escuti, C. Packham, and J. Codona, "The vector-APP: a broadband apodizing phase plate that yields complementary PSFs," in Society of Photo-Optical Instrumentation Engineers (SPIE) Conference Series, Society of Photo-Optical Instrumentation Engineers (SPIE) Conference Series 8450, Sept. 2012.

[22] G. Otten, F. Snik, M. Kenworthy, M. Miskiewicz, and M. Escuti, "The vector-Apodizing Phase Plate coronagraph," Applied Optics , 2014

[23] Y. Li, J. Kim, and M. J. Escuti, "Broadband orbital angular momentum manipulation using liquid crystal thin films," in Society of Photo-Optical Instrumentation Engineers (SPIE) Conference Series, Society of Photo-Optical Instrumentation Engineers (SPIE) Conference Series 8274, Feb. 2012.

[24] C. Oh and M. J. Escuti, "Achromatic diffraction from polarization gratings with high efficiency," Opt. Lett. 33, pp. 2287-2289, Oct 2008.

[25] R. K. Komanduri, K. F. Lawler, and M. J. Escuti, "Multi-twist retarders: broadband retardation control using self-aligning reactive liquid crystal layers," Opt. Express 21, pp. 404-420, Jan 2013.

[26] D. Mawet, L. Pueyo, A. Carlotti, B. Mennesson, E. Serabyn, and J. K. Wallace, "Ring-apodized Vortex Coronagraphs for Obscured Telescopes. I. Transmissive Ring Apodizers," ApJS 209, p. 7, Nov. 2013.

[27] A. Carlotti, L. Pueyo, and D. Mawet, "Apodized phase mask coronagraphs for arbitrary apertures. II. Comprehensive review of solutions for the vortex coronagraph," A\&SA 566, p. A31, June 2014. 
[28] E. Serabyn, D. Mawet, and R. Burruss, "An image of an exoplanet separated by two diffraction beamwidths from a star," Nature 464, pp. 1018-1020, Apr. 2010.

[29] O. Absil, J. Milli, D. Mawet, A.-M. Lagrange, J. Girard, G. Chauvin, A. Boccaletti, C. Delacroix, and J. Surdej, "Searching for companions down to $2 \mathrm{AU}$ from $\beta$ Pictoris using the L'-band AGPM coronagraph on. VLT/NACO," A 6 A 559, p. L12, Nov. 2013.

[30] D. Mawet, E. Serabyn, J. K. Wallace, and L. Pueyo, "Improved high-contrast imaging with on-axis telescopes using a multistage vortex coronagraph," Opt. Lett. 36, pp. 1506-1508, Apr 2011.

[31] E. Serabyn, J. K. Wallace, and D. Mawet, "Speckle-phase measurement in a tandem-vortex coronagraph," Appl. Opt. 50, pp. 5453-5456, Oct 2011.

[32] P. Riaud, D. Mawet, and A. Magette, "Instantaneous phase retrieval with the vector vortex coronagraph. Theoretical and optical implementation," A\&SA 545, p. A151, Sept. 2012.

[33] D. Mawet, "Annular Groove Phase Mask: An Achromatic Vortex Coronagraph Intended at Differential Polarimetric Imaging," in In the Spirit of Bernard Lyot: The Direct Detection of Planets and Circumstellar Disks in the 21st Century, June 2007. 